\title{
Analyzing the Anomalous Dipole Moment Type Couplings of Heavy Quarks with FCNC Interactions at the CLIC
}

\author{
A. T. Tasci, A. Senol, and C. Verep \\ Department of Physics, Kastamonu University, 37100 Kastamonu, Turkey \\ Correspondence should be addressed to A. T. Tasci; attasci@gmail.com
}

Received 24 October 2013; Revised 11 February 2014; Accepted 24 March 2014; Published 9 April 2014

Academic Editor: Alexey A. Petrov

Copyright ( 2014 A. T. Tasci et al. This is an open access article distributed under the Creative Commons Attribution License, which permits unrestricted use, distribution, and reproduction in any medium, provided the original work is properly cited. The publication of this article was funded by SCOAP S $^{3}$

\begin{abstract}
We examine both anomalous magnetic and dipole moment type couplings of a heavy quark via its single production with subsequent dominant standard model decay modes at the compact linear collider (CLIC). The signal and background cross sections are analyzed for heavy quark masses 600 and $700 \mathrm{GeV}$. We make the analysis to delimitate these couplings as well as to find the attainable integrated luminosities for $3 \sigma$ observation limit.
\end{abstract}

\section{Introduction}

Discovery of new particles performs a crucial role for physics beyond the standard model (SM) and may play a milestone role in the discovery of some open questions like the electroweak symmetry breaking [1-5], fermion mass spectrum hierarchies and mixing angle in quark/lepton sectors [6-10], $\mathrm{CP}$ violation, and flavor structure of standard theory [11-17]. The precise determination of heavy quark properties may present the existence of new physics. A heavy down-type quark $\left(b^{\prime}\right)$ with mass less than $645 \mathrm{GeV}$ and an up-type quark $\left(t^{\prime}\right)$ with mass less than $585 \mathrm{GeV}$ [18] are excluded at $95 \%$ confidence level from proton-proton collisions at $\sqrt{s}=8 \mathrm{TeV}$ ATLAS detector at the CERN large hadron collider.

Searching for new sources of CP violation beyond the SM is an attractive subject in particle physics, since it explains the asymmetry between matter and antimatter. $\mathrm{CP}$ violating anomalous flavor changing neutral current (FCNC) $t c Z / t c \gamma$ couplings have been considered in the literature before at hadron [19] and $e^{-} e^{+}[20,21]$ colliders. This type of FCNC interactions offer an ideal place to search for new physics. Due to the large mass values, heavy quarks have crucial advantage to new interactions originating at a higher scale as in top quark physics. Recently, anomalous FCNC $t$ quark couplings, such as $\operatorname{tqV}(q=u c, V=\gamma, Z, g)$, were experimentally restricted by some collaborations. For instance, the upper limits observed from $t q g$ vertices by ATLAS collaboration are $\kappa_{\text {ugt }} / \Lambda<6.9 \times 10^{-3} \mathrm{TeV}^{-1}$ and $\kappa_{\text {cgt }} / \Lambda<$ $1.6 \times 10^{-2} \mathrm{TeV}^{-1}$ assuming only one coupling is kept nonzero [22], while D0 set limits are $\kappa_{\text {tgu }} / \Lambda<0.013$ $\mathrm{TeV}^{-1}, \kappa_{\text {tgc }} / \Lambda<0.057 \mathrm{TeV}^{-1}[23]$ and $\mathrm{CDF}$ set limits are $\kappa_{\text {tug }} / \Lambda<0.018 \mathrm{TeV}^{-1}, \kappa_{\text {tcg }} / \Lambda<0.069 \mathrm{TeV}^{-1}$ [24]. Recent observed upper limits on the coupling strengths from CMS collaboration, which analyzed both gqt and $Z q t$ vertices probed simultaneously, are $\kappa_{\text {gut }} / \Lambda<0.10 \mathrm{TeV}^{-1}, \kappa_{\text {gct }} / \Lambda<$ $0.35 \mathrm{TeV}^{-1}, \kappa_{Z u t} / \Lambda<0.45 \mathrm{TeV}^{-1}$, and $\kappa_{Z c t} / \Lambda<2.27 \mathrm{TeV}^{-1}$ [25].

Serious contributions can be expected for the production of the heavy fermions, due to the anomalous magnetic moment type interactions. Phenomenological studies with these anomalous effects of these quarks have been performed on hadron colliders [26-31], on electron proton colliders $[32,33]$, and on linear colliders [34]. In this work, we study the production of single heavy $t^{\prime}$ quark at compact linear collider (CLIC) [35] via both anomalous magnetic and dipole moment type interactions. CLIC, a most popular proposed linear collider on $\mathrm{TeV}$ scale, would complete the LHC results by performing precision measurements to provide necessary information about some parameters of heavy quarks. The aim of this study is to delimitate the anomalous magnetic 


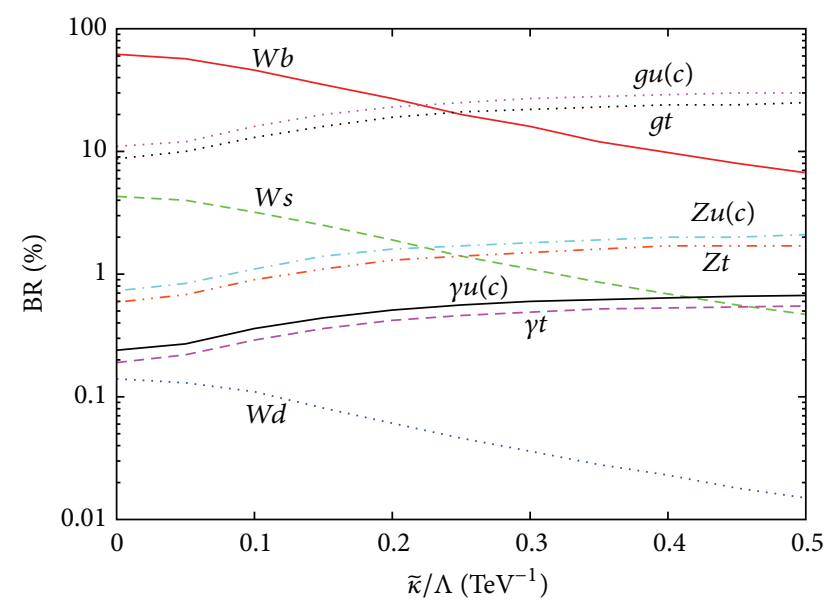

Figure 1: Branching ratios (\%) of all $t^{\prime}$ decay channels depending on $\tilde{\kappa} / \Lambda$ for $m_{t^{\prime}}=700 \mathrm{GeV}$.

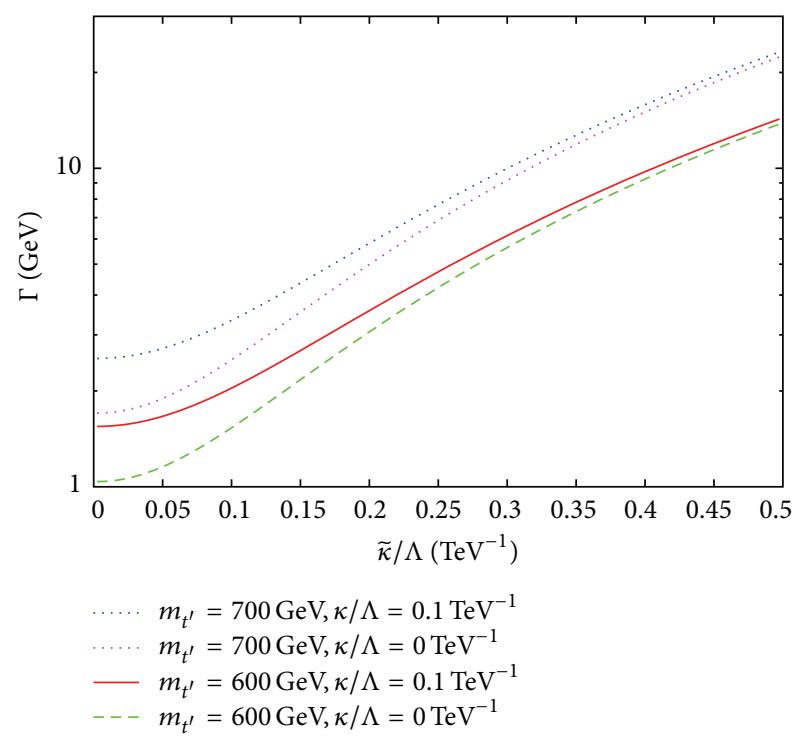

FIgURE 2: The total decay width of the $t^{\prime}$ quark as function of $\widetilde{\kappa} / \Lambda$.

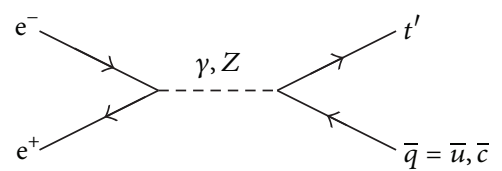

FIGURE 3: Feynman diagram for anomalous single production of $t^{\prime}$ quark in $e^{+} e^{-}$collision.

and dipole moment type couplings of $t^{\prime}$ quark from a detailed signal and background analysis including Monte Carlo simulation with the effects of initial state radiation (ISR) and beamstrahlung (BS) in the $e^{+} e^{-}$collisions.

\section{Single Production and Decay of $t^{\prime}$ Quark}

The interaction Lagrangian for $t^{\prime}$ quark within the SM is given by

$$
\begin{aligned}
L_{s}= & -g_{e} Q_{t^{\prime}} \bar{t}^{\prime} \gamma^{\mu} t^{\prime} A_{\mu} \\
& -g_{s} \bar{t}^{\prime} T^{a} \gamma^{\mu} t^{\prime} G_{\mu}^{a}
\end{aligned}
$$

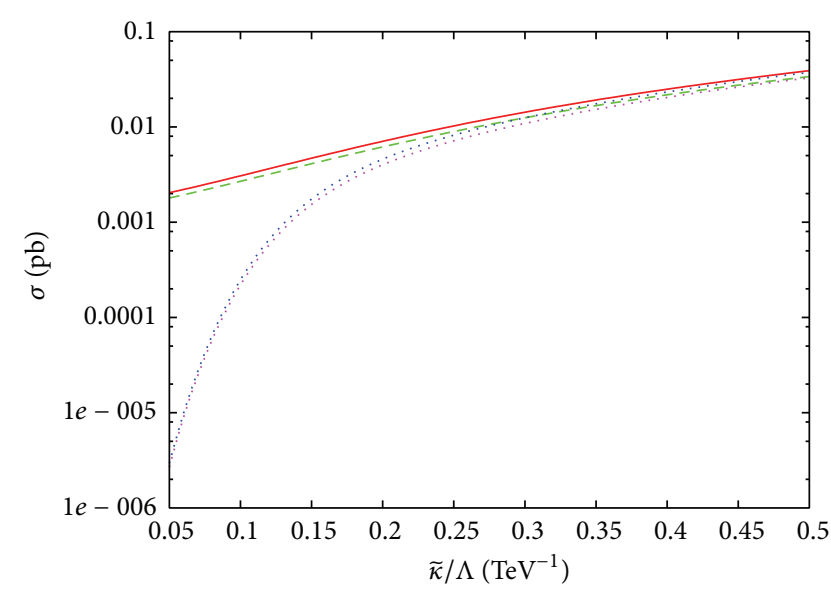

$$
\begin{aligned}
-m_{t^{\prime}} & =600 \mathrm{GeV}, \kappa / \Lambda=0.1 \mathrm{TeV}^{-1} \\
---m_{t^{\prime}} & =700 \mathrm{GeV}, \kappa / \Lambda=0.1 \mathrm{TeV}^{-1} \\
\cdots \cdots & m_{t^{\prime}}=600 \mathrm{GeV}, \kappa / \Lambda=0 \mathrm{TeV}^{-1} \\
\cdots \cdots m_{t^{\prime}} & =700 \mathrm{GeV}, \kappa / \Lambda=0 \mathrm{TeV}^{-1}
\end{aligned}
$$

Figure 4: The total cross sections at $\sqrt{s}=3 \mathrm{TeV}$ for the process $e^{+} e^{-} \rightarrow t^{\prime} \bar{q}(\bar{q}=\bar{u}, \bar{c})$ as function of $\widetilde{\kappa} / \Lambda$.

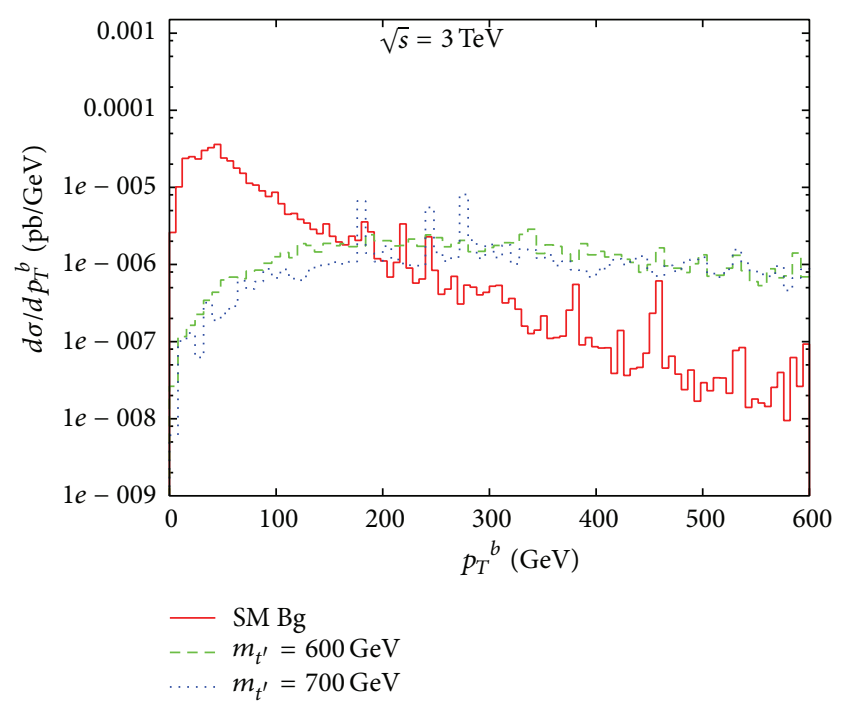

FIgURE 5: The differential cross section depending on the transverse momentum of the final state $b$ quark of process $e^{+} e^{-} \rightarrow W^{+} b \bar{q}(\bar{q}=$ $\bar{u}, \bar{c}$ ) for SM background (solid line) and signal with different mass values of $t^{\prime}$ quarks at $\sqrt{s}=3 \mathrm{TeV}$.

$$
\begin{aligned}
& -\frac{g_{e}}{2 s_{W} c_{W}} \bar{t}^{\prime} \gamma^{\mu}\left(g_{V}-g_{A} \gamma^{5}\right) t^{\prime} Z_{\mu}^{0} \\
& -\frac{g_{e}}{2 \sqrt{2} s_{W}} V_{t^{\prime}} q_{i} \bar{t}^{\prime} \gamma^{\mu}\left(1-\gamma^{5}\right) q_{i} W_{\mu}^{+}+\text {h.c. }
\end{aligned}
$$

where $A_{\mu}, G_{\mu}, Z_{\mu}^{0}$, and $W_{\mu}^{+}$are the vector fields for photon, gluon, $Z$ boson, and $W$ boson, respectively. $g_{e}$ is the electroweak coupling constant and $g_{s}$ is the strong coupling constant. $T^{a}$ are the Gell-Mann matrices; $Q_{t^{\prime}}$ is the electric charge of heavy quark $t^{\prime} \cdot g_{V}$ and $g_{A}$ are the vector and axial-vector type couplings of the neutral weak current with 


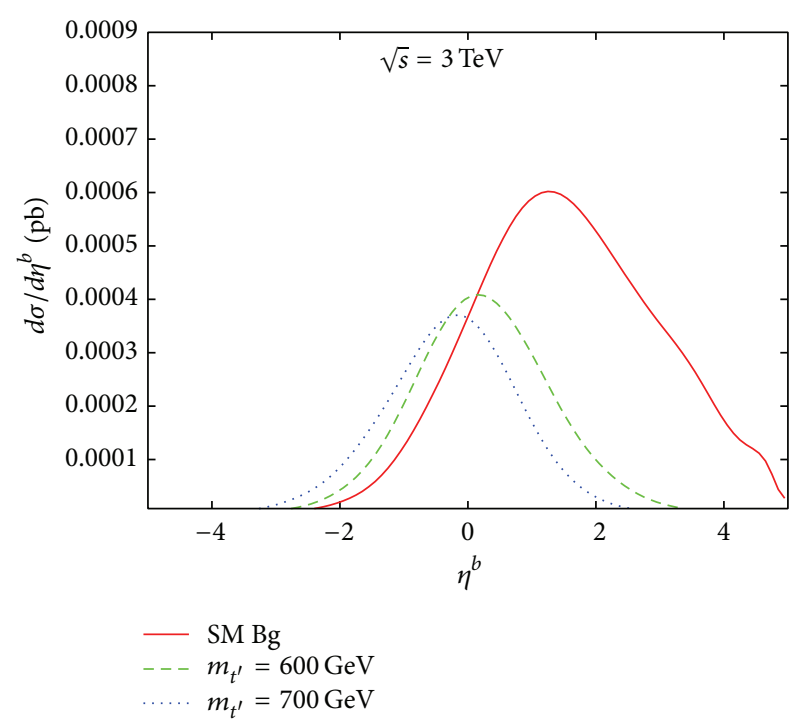

FIgURE 6: The rapidity distribution of the final state $b$ quark at $\sqrt{s}=$ $3 \mathrm{TeV}$ for the process $e^{+} e^{-} \rightarrow W^{+} b \bar{q}(\bar{q}=\bar{u}, \bar{c})$.

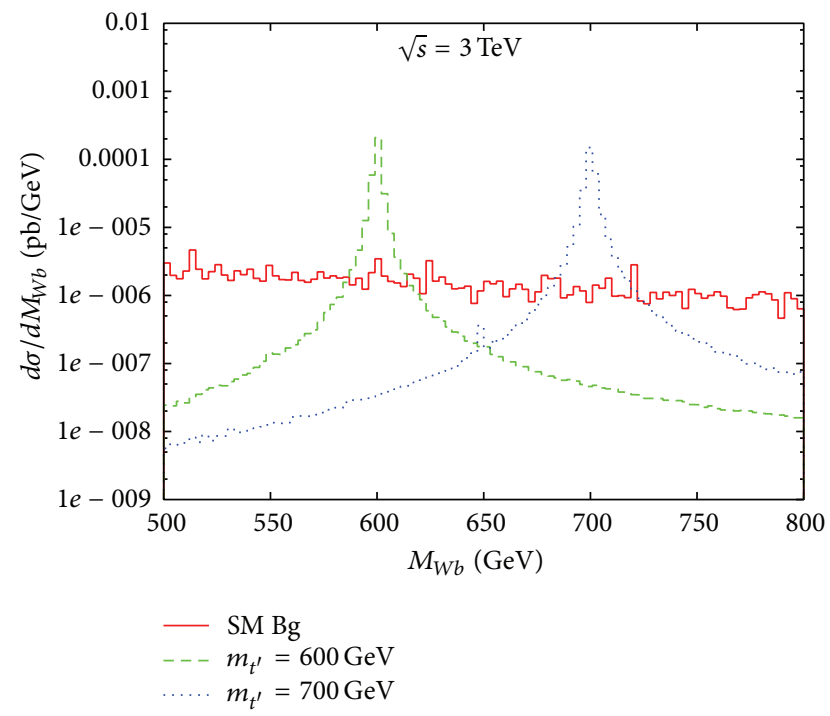

Figure 7: The invariant mass distribution of the final state $W b$ system for SM background (solid line) and signal from $t^{\prime}$ decay for $m_{t^{\prime}}=600 \mathrm{GeV}$ (dashed line) and $m_{t^{\prime}}=700 \mathrm{GeV}$ (dot-dashed line) at $\sqrt{s}=3 \mathrm{TeV}$.

$t^{\prime}$ quark; $\theta_{W}$ is the weak mixing angle, $s_{W}=\sin \theta_{W}$ and $c_{W}=\cos \theta_{W} \cdot V_{t^{\prime} q}$ denotes the elements of extended $4 \times 4$ CKM mixing matrix which are constrained by flavor physics.

The anomalous magnetic and dipole moment type interactions among heavy quark $t^{\prime}$, ordinary quarks $q$, and the neutral gauge bosons $V=\gamma, Z, g$ can be described by an effective Lagrangian which contains the anomalous magnetic and dipole moment type couplings given by

$$
\begin{aligned}
L_{a}^{\prime}= & \sum_{q_{i}=u, c, t} Q_{q_{i}} \frac{g_{e}}{\Lambda} \bar{t}^{\prime} \sigma_{\mu \nu}\left(\kappa_{\gamma}^{q_{i}}-i \widetilde{\kappa}_{\gamma}^{q_{i}} \gamma_{5}\right) q_{i} F^{\mu \nu} \\
& +\sum_{q_{i}=u, c, t} \frac{g_{e}}{2 \Lambda s_{W} c_{W}} \bar{t}^{\prime} \sigma_{\mu \nu}\left(\kappa_{Z}^{q_{i}}-i \widetilde{\kappa}_{Z}^{q_{i}} \gamma_{5}\right) q_{i} Z^{\mu \nu}
\end{aligned}
$$

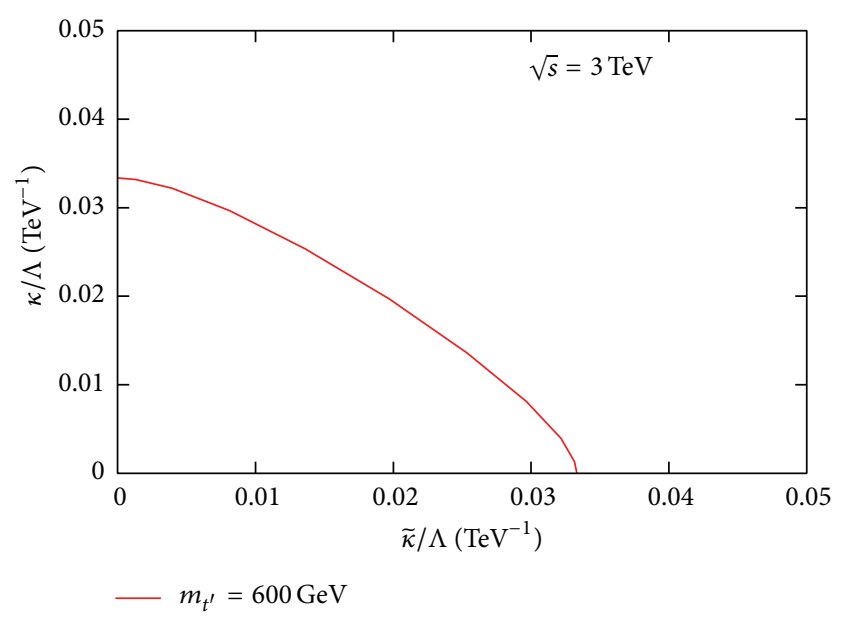

FIgURE 8: The $3 \sigma$ contour plot for the anomalous couplings reachable at $\sqrt{s}=3 \mathrm{TeV}$ with $L_{\text {int }}=5.9 \times 10^{5} \mathrm{pb}^{-1}$ for $m_{t^{\prime}}=600 \mathrm{GeV}$.

TABLE 1: Main parameters of the CLIC.

\begin{tabular}{lc}
\hline Parameters & CLIC \\
\hline$E_{\mathrm{cm}}(\sqrt{s}) \mathrm{TeV}$ & 3 \\
$L\left(10^{34} \mathrm{~cm}^{-2} \mathrm{~s}^{-1}\right)$ & 5.9 \\
$N\left(10^{10}\right)$ & 0.372 \\
$\sigma_{x}(\mathrm{~nm})$ & 45 \\
$\sigma_{y}(\mathrm{~nm})$ & 1 \\
$\sigma_{z}(\mu \mathrm{m})$ & 44 \\
\hline
\end{tabular}

Here, $N$ is the number of particles in bunch; $\sigma_{x}$ and $\sigma_{y}$ are beam sizes; and $\sigma_{z}$ is the bunch length.

$$
+\sum_{q_{i}=u, c, t} \frac{g_{s}}{2 \Lambda} \bar{t}^{\prime} \sigma_{\mu \nu}\left(\kappa_{g}^{q_{i}}-i \widetilde{\kappa}_{g}^{q_{i}} \gamma_{5}\right) T^{a} q_{i} G_{a}^{\mu \nu}+\text { h.c. }
$$

where $F^{\mu \nu}, Z^{\mu \nu}$, and $G^{\mu \nu}$ are the field strength tensors of the gauge bosons; $\sigma_{\mu \nu}=i\left(\gamma_{\mu} \gamma_{\nu}-\gamma_{\nu} \gamma_{\mu}\right) / 2 ; Q_{q_{i}}$ is the electric charge of the quark $(q) \cdot \kappa_{\gamma}\left(\widetilde{\kappa}_{\gamma}\right), \kappa_{Z}\left(\widetilde{\kappa}_{Z}\right)$ and $\kappa_{g}\left(\widetilde{\kappa}_{g}\right)$ are the anomalous magnetic (dipole) moment type couplings with photon, $Z$ boson, and gluon, respectively. Note that $\widetilde{\kappa}$ s are $C P$ violating and $\Lambda$ is the cutoff scale of new interactions and we assume $\kappa_{\gamma}=\kappa_{Z}=\kappa_{g}=\kappa$ and $\widetilde{\kappa}_{\gamma}=\widetilde{\kappa}_{Z}=\widetilde{\kappa}_{g}=\widetilde{\kappa}$.

$\mathrm{CP}$ violating flavor changing neutral current processes within the SM with the $b^{\prime}$ and $t^{\prime}$ quarks are analyzed by constructing and employing global unique fit of the unitary $4 \times 4 \mathrm{CKM}$ mass mixing matrix at $m_{t^{\prime}}=600$ and $700 \mathrm{GeV}$ separately, in [36]. In our calculations we use this parameterization for values of the $4 \times 4 \mathrm{CKM}$ matrix elements and we assume $m_{t^{\prime}}>m_{b^{\prime}}$ with a mass splitting of $m_{t^{\prime}}-m_{b^{\prime}} \approx 50 \mathrm{GeV}$. We implement the related interaction vertices, given in the effective Lagrangian, into the tree level event generator CompHEP package [37] for numerical calculations. In Figure 1, branching ratios (BR) dependence on $\widetilde{\kappa} / \Lambda$ for SM decay channels $(W d(s, b))$ and anomalous decay channels $(V u(c, t))$ of $t^{\prime}$ quark which are calculated by using Lagrangians (1) and (2) are given for $m_{t^{\prime}}=700 \mathrm{GeV}$ and $\kappa / \Lambda=0.1 \mathrm{TeV}^{-1}$. As seen from this figure, SM $t^{\prime}$ decay channel, $t^{\prime} \rightarrow W b$, is dominant for $\widetilde{\kappa} / \Lambda$ less than 0.2 


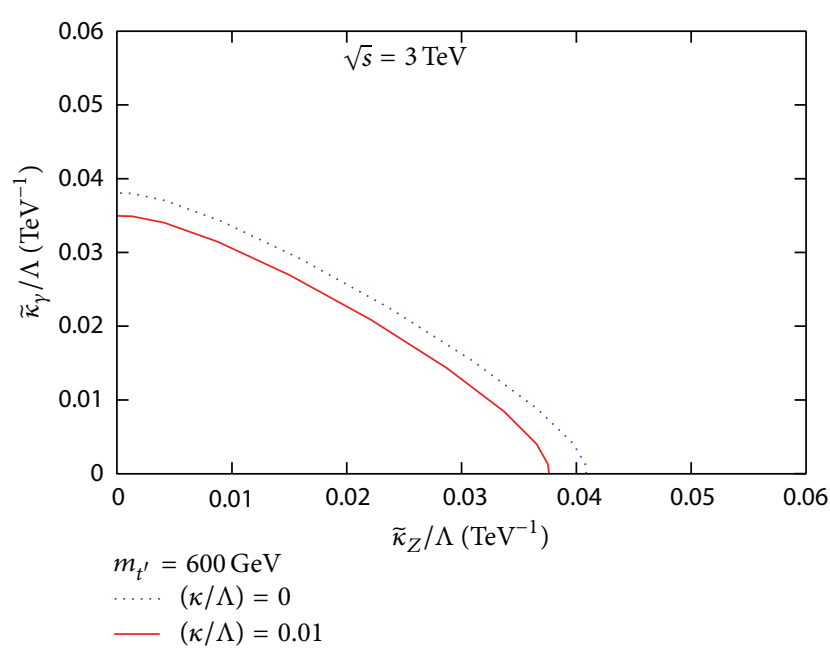

(a)

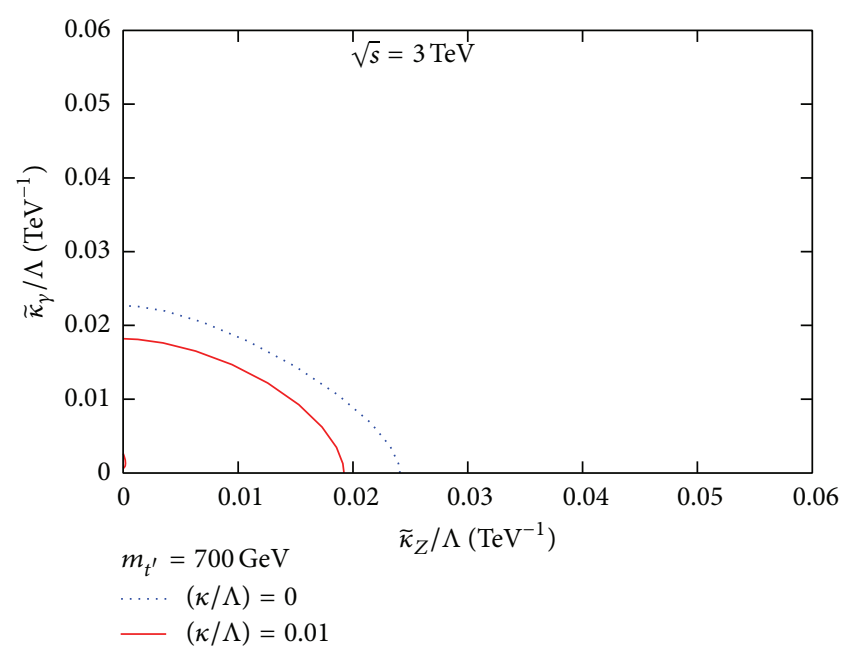

(b)

Figure 9: The $3 \sigma$ contour plot for the anomalous dipole moment type couplings reachable at (a) $m_{t^{\prime}}=600 \mathrm{GeV}$ and (b) $m_{t^{\prime}}=700 \mathrm{GeV}$.

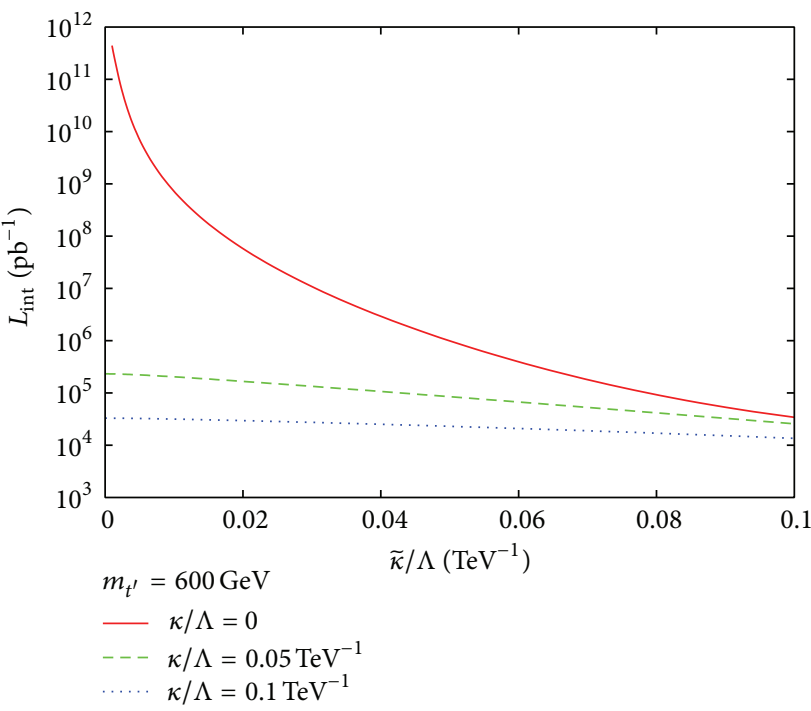

(a)

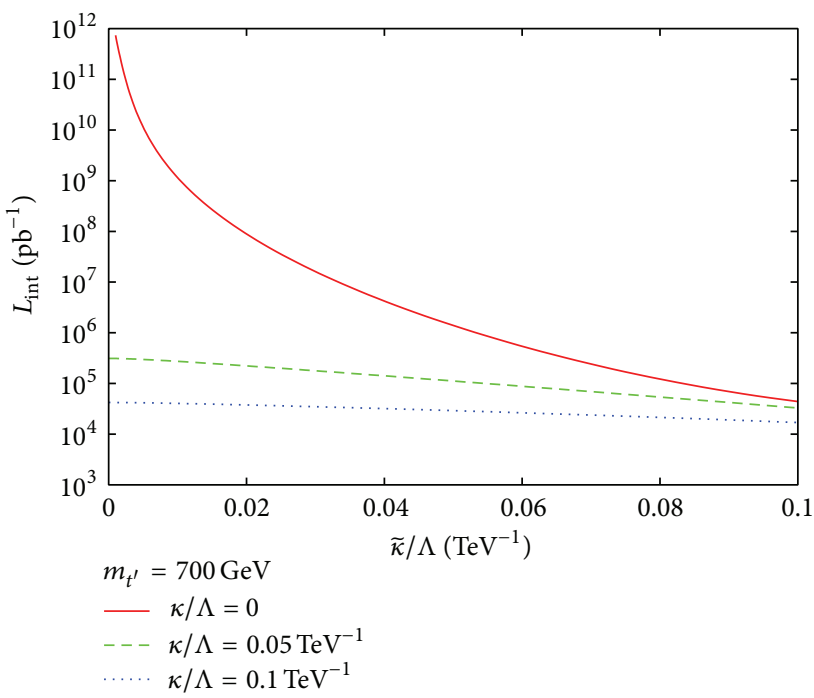

(b)

Figure 10: The attainable integrated luminosity for $3 \sigma$ observation limit for (a) $m_{t^{\prime}}=600 \mathrm{GeV}$ and (b) $m_{t^{\prime}}=700 \mathrm{GeV}$ for $\sqrt{s}=3 \mathrm{TeV}$ depending on anomalous dipole moment type couplings.

changing around $27 \%-63 \% \mathrm{BR}$. Total decay widths of $t^{\prime}$ quark dependence on $\widetilde{\kappa} / \Lambda$ are given in Figure 2 for $m_{t^{\prime}}=600$ and $700 \mathrm{GeV}$ with $\kappa / \Lambda=0$ and $0.1 \mathrm{TeV}^{-1}$.

The contributing tree level Feynman Diagram for the anomalous single production of $t^{\prime}$ quark in $e^{+} e^{-}$collision is shown in Figure 3. In Figure 4, the total cross sections for single production of $t^{\prime}$ quark are plotted at collision center of mass energy of $3 \mathrm{TeV}$ with respect to $\widetilde{\kappa} / \Lambda$ for $m_{t^{\prime}}=600$ and $700 \mathrm{GeV}$ with $\kappa / \Lambda=0$ and $0.1 \mathrm{TeV}^{-1}$. Initial state radiation (ISR) and beamstrahlung (BS) are specific features of the linear colliders. We take the beam parameters for the CLIC given in Table 1, when calculating the ISR and BS effects. Hereafter, in all our numerical calculations we take into account ISR + BS effects.

\section{Signal and Background Analysis}

The signal process of single production of $t^{\prime}$ quark including the dominant SM decay mode over anomalous decay is $e^{+} e^{-} \rightarrow t^{\prime} \bar{q}_{i} \rightarrow W^{+} b \bar{q}_{i}$, where $\bar{q}_{i}=\bar{u}, \bar{c}$. The dominant source of SM background process is $e^{+} e^{-} W^{+} b \bar{q}_{i}$ for the corresponding signal processes.

In the transverse momentum, rapidity, and invariant mass distributions analysis, we assume $\tilde{\kappa} / \Lambda=\kappa / \Lambda=0.1 \mathrm{TeV}^{-1}$. In Figure 5, the transverse momentum $\left(p_{T}\right)$ distributions of the final state $b$ quark for signal and background are shown for CLIC energy. We applied a $p_{T}$ cut of $p_{T}>50 \mathrm{GeV}$ to reduce the background, comparing the signal $p_{T}$ distribution of $b$ quark with that of the corresponding background. 
TABLE 2: The signal and background cross sections and signal statistical significance (SS) by taking $\kappa / \Lambda=0 \mathrm{TeV}^{-1}$ for the $\mathrm{CLIC}$ at $\sqrt{s}=3 \mathrm{TeV}$ with integrated luminosity of $5.9 \times 10^{5} \mathrm{pb}^{-1}$.

\begin{tabular}{lccccc}
\hline$m_{t^{\prime}}(\mathrm{GeV})$ & \multicolumn{3}{c}{$/ \Lambda=0.1 \mathrm{TeV}^{-1}$} & \multicolumn{3}{c}{$\tilde{\kappa} / \Lambda=0.01 \mathrm{TeV}^{-1}$} \\
& $\sigma_{S}(\mathrm{fb})$ & $\sigma_{B}(\mathrm{fb})$ & $\mathrm{SS}$ & $\sigma_{S}(\mathrm{fb})$ & $\sigma_{B}(\mathrm{fb})$ \\
\hline 600 & 3.01 & $8.92 \times 10^{-3}$ & 19.72 & $1.50 \times 10^{-2}$ & $8.92 \times 10^{-3}$ \\
700 & 2.63 & $1.14 \times 10^{-2}$ & 18.43 & $1.31 \times 10^{-2}$ & $1.14 \times 10^{-2}$ \\
\hline
\end{tabular}

In Figure 6, we plot the rapidity distributions of final state $b$ quark in signal and background processes. According to these figures, the cut $\left|\eta^{b}\right|<2.5$ can be applied to suppress the background while the signal remains almost unchanged.

In Figure 7, the invariant mass distributions for the $W^{+} b$ system in the final state are plotted. From these figures, we can see that the signal has a peak around mass of $t^{\prime}$ quark over the background.

In Table 2, we calculate cross sections of the signal and background and the statistical significance (SS) to discuss the observability of 600 and $700 \mathrm{GeV} t^{\prime}$ quark for $\widetilde{\kappa} / \Lambda=0.1$ and $0.01 \mathrm{TeV}^{-1}$ by taking $\kappa / \Lambda=0 \mathrm{TeV}^{-1}$ at CLIC. The SS of the signal is obtained by using the following formula:

$$
\mathrm{SS}=\left(\frac{\sigma_{S}}{\sqrt{\sigma_{S}+\sigma_{B}}}\right) \sqrt{\mathrm{BR}\left(W \longrightarrow l \nu_{l}\right) \cdot L_{\mathrm{int}}}
$$

where $\sigma_{S}$ is the signal and $\sigma_{B}$ is the background cross sections for the $e^{+} e^{-} \rightarrow t^{\prime} \bar{q}_{i} \rightarrow W^{+} b \bar{q}_{i}$ process, respectively, and $l=e, \mu$. We take into account finite energy resolution of the detectors for realistic analysis. In our numerical calculations we use the mass bin width $\Delta m=\max (2 \Gamma, \delta m)$ to count signal and background events with the mass resolution $\delta m$. The mentioned $p_{T}$ and $\eta$ cuts are applied assuming the integrated luminosity given in Table 1.

After this point, we will focus on limiting the anomalous magnetic and dipole moment type couplings. Firstly, in Figure 8 , we present the $3 \sigma$ contour plot for $\widetilde{\kappa} / \Lambda-\kappa / \Lambda$ plane at $\sqrt{s}=3 \mathrm{TeV}$ with $m_{t^{\prime}}=600 \mathrm{GeV}$. According to these figures, the lower limits of $\kappa / \Lambda$ and $\tilde{\kappa} / \Lambda$ are about $0.033 \mathrm{TeV}^{-1}$ at the CLIC energy.

To analyze the case of $\widetilde{\kappa}_{\gamma} / \Lambda \neq \widetilde{\kappa}_{Z} / \Lambda$, the $3 \sigma$ contour plots for the anomalous couplings in the $\widetilde{\kappa}_{Z} / \Lambda-\widetilde{\kappa}_{\gamma} / \Lambda$ plane are presented in Figure 9 at $\sqrt{s}=3 \mathrm{TeV}$ with (a) $m_{t^{\prime}}=600 \mathrm{GeV}$ and (b) $m_{t^{\prime}}=700 \mathrm{GeV}$ by taking into account different values of $\kappa / \Lambda$. According to these figures the lower limits of $\widetilde{\kappa}_{\gamma} / \Lambda$ and $\widetilde{\kappa}_{Z} / \Lambda$ are about $0.038 \mathrm{TeV}^{-1}$ for $m_{t^{\prime}}=600 \mathrm{GeV}$ and $0.019 \mathrm{TeV}^{-1}$ for $m_{t^{\prime}}=700 \mathrm{GeV}$ with $\kappa / \Lambda=0.01 \mathrm{TeV}^{-1}$. In Figures 8 and 9 , allowed parameter space area of $t^{\prime}$ quark is above the lines.

We plot the lowest necessary luminosities with $3 \sigma$ observation limits for (a) $m_{t^{\prime}}=600 \mathrm{GeV}$ and (b) $m_{t^{\prime}}=700 \mathrm{GeV}$ at $\sqrt{s}=3 \mathrm{TeV}$ depending on anomalous couplings in Figure 10. In the case of $\kappa / \Lambda=\widetilde{\kappa} / \Lambda=0.1 \mathrm{TeV}^{-1}$, it is seen that from these figures, $t^{\prime}$ quarks with masses 600 and $700 \mathrm{GeV}$ can be observed at $3 \sigma$ observation limit with lowest integrated luminosity at the order of $10^{4} \mathrm{pb}^{-1}$ at CLIC.

\section{Conclusion}

The anomalous FCNC interactions of heavy quarks could be important for some parameter regions due to the expected large masses. The sensitivity to the anomalous couplings $(\kappa$, $\widetilde{\kappa})$ and $\left(\widetilde{\kappa}_{\gamma}, \widetilde{\kappa}_{Z}\right)$ can be obtained for $m_{t^{\prime}}=600 \mathrm{GeV}$ about $(0.033,0.033)$ and $(0.035,0.038)$ for $\kappa=0.01$, and for $m_{t^{\prime}}=$ $700 \mathrm{GeV}\left(\widetilde{\kappa}_{\gamma}, \widetilde{\kappa}_{Z}\right)$ values can be obtained about $(0.019,0.0195)$ for $\kappa=0.01$ with $\Lambda=1 \mathrm{TeV}$. We also find the lowest necessary luminosity limit values at the order of $10^{4} \mathrm{pb}^{-1}$ for CLIC.

\section{Conflict of Interests}

The authors declare that there is no conflict of interests regarding the publication of this paper.

\section{Acknowledgments}

This research has been partially supported by Kastamonu University Scientific Research Projects Coordination Department under the Grant no. KUBAP-03/2012-01.

\section{References}

[1] B. Holdom, "Heavy quarks and electroweak symmetry breaking," Physical Review Letters, vol. 57, no. 20, pp. 2496-2499, 1986.

[2] B. Holdom, "Erratum to "Heavy quarks and electroweaksymmetry breaking'"' Physical Review Letters, vol. 58, no. 2, p. 177, 1987.

[3] C. T. Hill, M. A. Luty, and E. A. Paschos, "Electroweak symmetry breaking by fourth-generation condensates and the neutrino spectrum," Physical Review D, vol. 43, no. 9, pp. 30113025, 1991.

[4] T. Elliott and S. F. King, "Heavy quark condensates from dynamically broken flavour symmetry," Physics Letters B, vol. 283, no. 3-4, pp. 371-378, 1992.

[5] P. Q. Hung and C. Xiong, "Dynamical electroweak symmetry breaking with a heavy fourth generation," Nuclear Physics B, vol. 848, no. 2, pp. 288-302, 2011.

[6] B. Holdom, "The discovery of the fourth family at the LHC: wat if?" Journal of High Energy Physics, vol. 2006, no. 8, article 076, 2006.

[7] P. Q. Hung and M. Sher, "Experimental constraints on fourth generation quark masses," Physical Review D, vol. 77, no. 3, Article ID 037302, 2008.

[8] P. Q. Hung and C. Xiong, "Implication of a quasi fixed point with a heavy fourth generation: the emergence of a TeV-scale physical cutoff," Physics Letters B, vol. 694, no. 4-5, pp. 430-434, 2011. 
[9] P. Q. Hung and C. Xiong, "Renormalization group fixed point with a fourth generation: Higgs-induced bound states and condensates," Nuclear Physics B, vol. 847, no. 1, pp. 160-178, 2011.

[10] O. Cakir, A. Senol, and A. T. Tasci, "Single production of fourthfamily $\mathrm{t}$ ' quarks at the CERN large hadron electron collider," Europhysics Letters, vol. 88, no. 1, Article ID 11002, 2009.

[11] W.-S. Hou and C.-Y. Ma, "Flavor and CP violation with fourth generations revisited ", Physical Review D, vol. 82, Article ID 036002, 2010.

[12] S. Bar-Shalom, D. Oaknin, and A. Soni, "Extended FriedbergLee hidden symmetries, quark masses, and CP violation with four generations," Physical Review D, vol. 80, no. 1, Article ID 015011, 2009.

[13] A. J. Buras, B. Duling, T. Feldmann, T. Heidsieck, C. Promberger, and S. Recksiegel, "Patterns of flavour violation in the presence of a fourth generation of quarks and leptons," Journal of High Energy Physics, vol. 2010, no. 9, article 106, 2010.

[14] A. Soni, A. K. Alok, A. Giri, R. Mohanta, and S. Nandi, "The fourth family: a simple explanation for the observed pattern of anomalies in $B-C P$ asymmetries," Physics Letters $B$, vol. 683, no. 4-5, pp. 302-305, 2010.

[15] O. Eberhardt, A. Lenz, and J. Rohrwild, "Less space for a new family of fermions," Physical Review D, vol. 82, no. 9, Article ID 095006, 2010.

[16] A. Soni, A. K. Alok, A. Giri, R. Mohanta, and S. Nandi, "Standard model with four generations: selected implications for rare B and K decays," Physical Review D, vol. 82, no. 3, Article ID 033009, 2010.

[17] A. K. Alok, A. Dighe, and D. London, "Constraints on the fourgeneration quark mixing matrix from a fit to flavor-physics data," Physical Review D, vol. 83, no. 7, Article ID 073008, 2011.

[18] The ATLAS collaboration, ATLAS-CONF-2013-056.

[19] T. Han, K. Whisnant, B.-L. Young, and X. Zhang, “Top-quark decay via the anomalous coupling t⿳亠 $\gamma$ at hadron colliders," Physical Review D, vol. 55, no. 11, pp. 7241-7248, 1997.

[20] T. Han and J. L. Hewett, "Top-charm associated production in high energy $e^{+} e^{-}$collisions ," Physical Review D, vol. 60, Article ID 074015, 1999.

[21] A. T. Alan, A. Senol, and A. T. Tasci, "CP violating asymmetries in the flavour changing single top quark production," Journal of Physics G, vol. 29, no. 2, pp. 279-284, 2003.

[22] G. Aad, B. Abbott, J. Abdallah et al., "Search for FCNC single top-quark production at $\sqrt{s}=7 \mathrm{TeV}$ with the ATLAS detector," Physics Letters B, vol. 712, no. 4-5, p. 351, 2012.

[23] V. M. Abazov, B. Abbott, M. Abolins et al., "Search for flavor changing neutral currents via quark-gluon couplings in single top quark production using $2.3 \mathrm{fb}^{-1}$ of $p \bar{p}$ collisions," Physics Letters B, vol. 693, p. 81, 2010.

[24] T. Aaltonen, J. Adelman, T. Akimoto et al., "Search for topquark production via flavor-changing neutral currents in $W+$ 1 jet events at CDF"' Physical Review Letters, vol. 102, Article ID 151801, 2009.

[25] E. Yazgan, "Flavor changing neutral currents in top quark production and decay," http://arxiv.org/abs/1312.5435.

[26] E. Arik, O. Çakir, and S. Sultansoy, "Anomalous single production of the fourth-SM-family quarks decaying into a light scalar boson at the Tevatron," Europhysics Letters, vol. 62, no. 3, pp. 332-335, 2003.

[27] E. Arik, O. Cakir, and S. Sultansoy, "Anomalous single production of the fourth SM family quarks at the Fermilab Tevatron," Physical Review D, vol. 67, no. 3, Article ID 035002, 2003.
[28] İ. T. Çakır, H. Duran Yıldız, O. Çakır, and G. Ünel, "Anomalous resonant production of the fourth-family up-type quarks at the LHC," Physical Review D, vol. 80, Article ID 095009, 2009.

[29] R. Çiftçi, "Anomalous single production of the fourth generation quarks at the CERN LHC," Physical Review D, vol. 78, Article ID 075018, 2008.

[30] M. Sahin, S. Sultansoy, and S. Turkoz, "Searching for the fourth family quarks through anomalous decays," Physical Review D, vol. 82, no. 5, Article ID 051503, 2010.

[31] O. Cakir, I. T. Cakir, A. Senol, and A. T. Tasci, "Anomalous single production of fourth family up-type quark associated with neutral gauge bosons at the LHC," Journal of Physics G, vol. 39, Article ID 055005, 2012.

[32] A. T. Alan, A. Senol, and O. Çakir, "Anomalous production of fourth-family up-quarks at future lepton hadron colliders," Europhysics Letters, vol. 66, no. 5, pp. 657-660, 2004.

[33] R. Çiftçi and A. K. Ciftci, "A comperative study of the anomalous single production of the fourth generation quarks at ep and gamma-p colliders," http://arxiv.org/abs/0904.4489.

[34] A. Senol, A. T. Tasci, and F. Ustabas, "Anomalous single production of fourth generation t' quarks at ILC and CLIC," Nuclear Physics B, vol. 851, no. 2, pp. 289-297, 2011.

[35] L. Linssen, A. Miyamoto, M. Stanitzki, and H. Weerts, "Physics and detectors at CLIC: CLIC conceptual design report," http://arxiv.org/abs/1202.5940.

[36] G. Eilam, B. Melic, and J. Trampetic, " $C P$ violation and the fourth generation,” Physical Review D, vol. 80, Article ID 116003, 2009.

[37] A. Pukhov, E. Boos, M. Dubinin et al., "CompHEP—a package for evaluation of Feynman diagrams and integration over multi-particle phasespace. User's manual for version 33," http://arxiv.org/abs/hep-ph/9908288. 

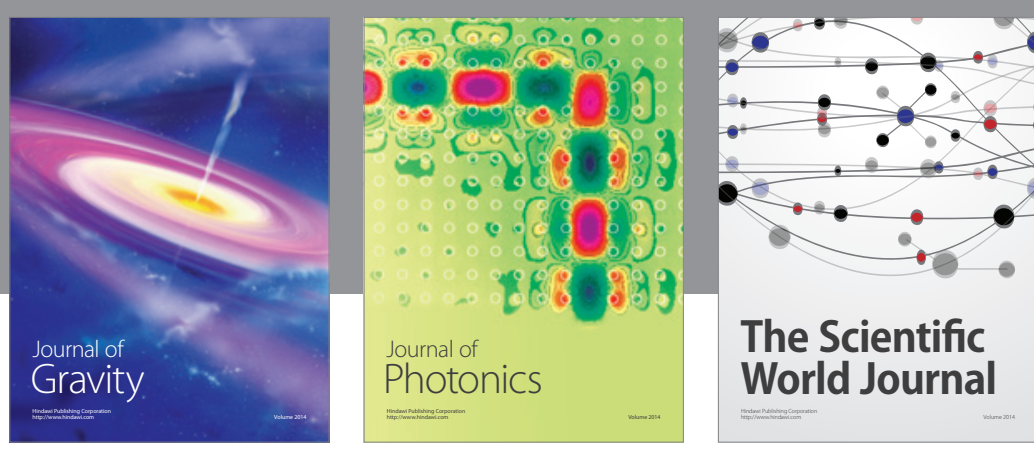

The Scientific World Journal
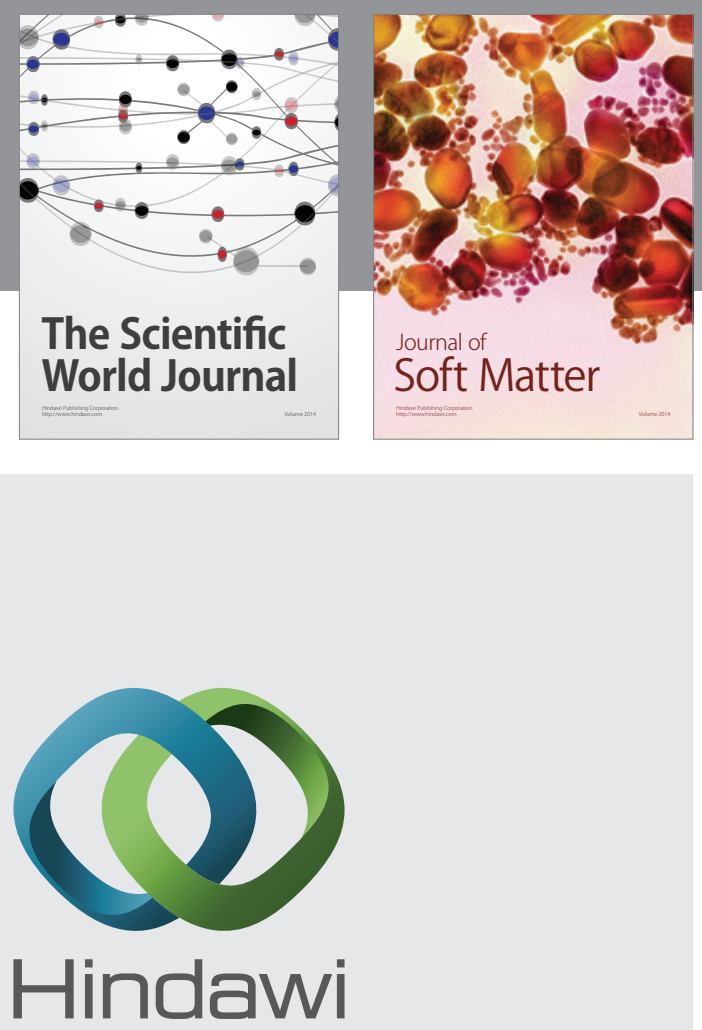

Submit your manuscripts at

http://www.hindawi.com

nternational Journal of

Statistical Mechanics
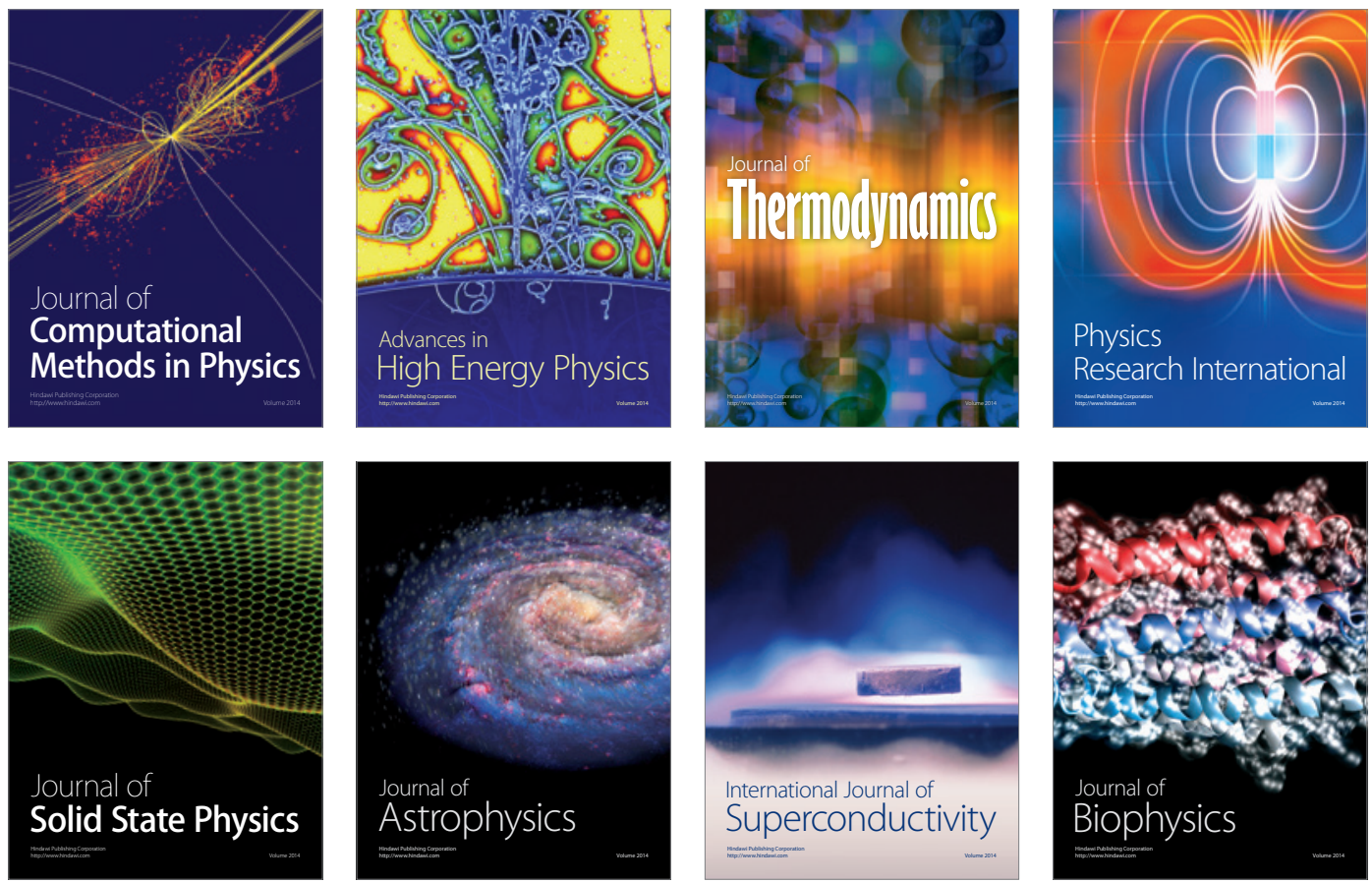
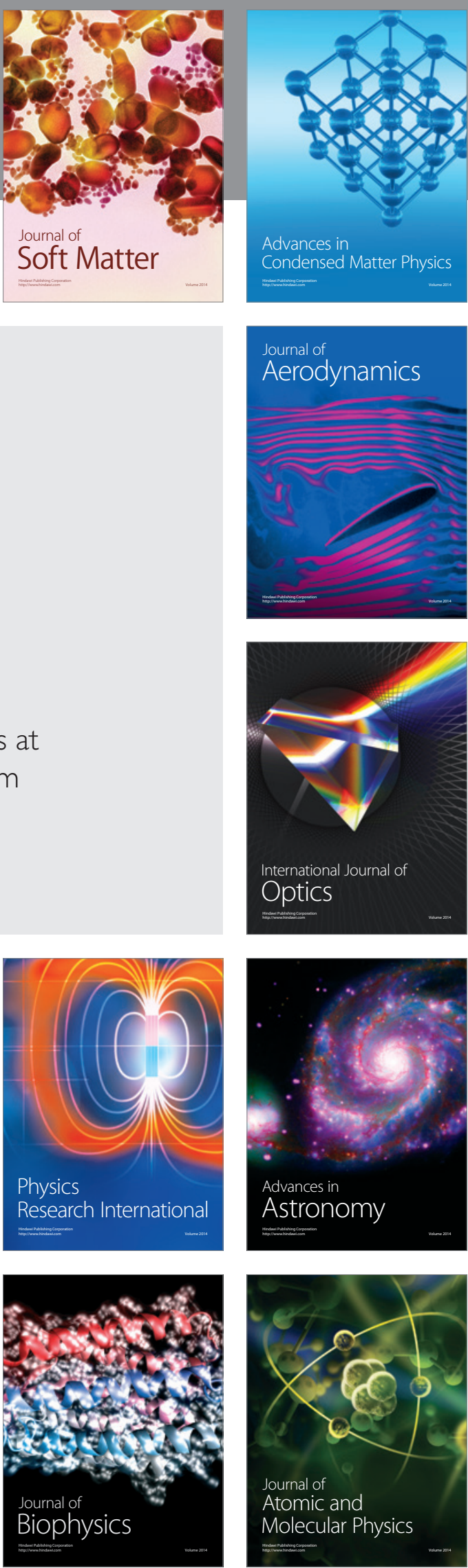